\title{
A CHOICE REACTION TIME ANALYSIS OF SPATIAL FREQUENCY DISCRIMINATION
}

\author{
Mark W. Greenlee and Bruno G. Breitmeyer* \\ Neurologische Universitätsklinik mit Abteilung für Neurophysiologie, Hansastrasse 9, \\ 7800 Freiburg, F.R.G.
}

(Received 1 June 1988; in revised form 7 March 1989)

\begin{abstract}
Simple reaction time to the onset of sinewave gratings was measured as a function of spatial frequency in two observers. These results are compared to the choice reaction time required for the observer to correctly discriminate the spatial frequency of two gratings flashed sequentially. Grating contrast was either 0.75 or 1.5 logarithmic units above the detection threshold for each spatial frequency tested. The spatial phase and contrast of the reference and test gratings were varied from trial to trial by small random amounts to eliminate fixed cues other than the difference frequency. The spatial frequency difference between the reference and test grating was either $0.125,0.25$ or 0.5 octave. As has been earlier reported, simple reaction time increases with increasing spatial frequency. Contrary to this, choice reaction time first increases (up to $4 \mathrm{c} / \mathrm{deg}$ ) and then decreases. We derived the time required by the observer to make a spatial frequency judgement by subtracting the simple reaction time from the choice reaction time for a given spatial frequency and contrast. The maximum decision time occurs in the medium spatial frequency range (between 1 and $4 \mathrm{c} / \mathrm{deg}$ ), at which frequencies we are most sensitive. The time required to make a correct spatial-frequency discrimination decreases with increasing spatial-frequency difference. The decision time is. however, fairly invariant over a large range of suprathreshold contrast levels. The findings suggest that the decision time for spatial frequency discrimination increases with the number of mechanisms involved.
\end{abstract}

Spatial-frequency discrimination Simple reaction time Choice reaction time Gratings Contrast

\section{INTRODUCTION}

Reaction time measurement has long been used as a means of exploring perceptual latency (for reviews of the earlier work see Johnson, 1923 and Teichner, 1953). The response latency to the onset of sinusoidal gratings has been shown to increase with increasing spatial frequency. This result has been interpreted as evidence for two parallel pathways in human vision, one quickly transmitting low spatial frequency, transient information, and the other more slowly transmitting high spatial frequency information (Breitmeyer, 1975; Lupp, Hauske \& Wolf, 1976; Vassilev \& Mitov, 1976; Harwerth \& Levi, 1978). Rudd (1988) has recently modelled the original data of Breitmeyer and found that reaction time increases linearly with the square of spatial frequency. This implies that temporal integration is inversely related to the spatial summation area, i.e. large receptive fields have a shorter integration time (and thus a shorter latency) than small receptive fields.

*Present address: Department of Psychology, University of Houston, Houston, TX 77004, U.S.A.
Earlier studies have concentrated on the dependency of simple reaction time on spatial frequency, contrast and exposure duration and have related these results to neurophysiological findings concerned with the conduction velocities and temporal characteristics of the so-called transient and sustained visual pathways (see Breitmeyer, 1984, for a review). The discrimination of two gratings having the same orientation, contrast and exposure duration but varying slightly in their spatial frequency has long been of interest, as spatial frequency discrimination is thought to reflect the action of mechanisms in the visual system scaled to different sizes (Campbell, Nachmias \& Jukes, 1970; Hirsch \& Hylton, 1982; Wilson \& Gelb, 1984). Although first thought to be a constant fraction of base spatial frequency (Campbell et al., 1970), Hirsch and Hylton (1982; and independently Yager \& Richter, 1982) showed that the Weber fraction for spatial frequency discrimination $(\Delta f / f)$ varied nonmonotonically with spatial frequency. Wilson and Gelb (1984) later modelled the data of Hirsch and Hylton using their modified line-element theory of spatial vision. They found that the fluctuations in spa- 
tial frequency discrimination at different reference spatial frequencies is in line with the concept of a limited number of channels discretely spaced in the spatial frequency domain.

We sought to unite these two areas in the literature, reaction time measurement and spatial frequency discrimination, by measuring the response latency occurring when a human observer was asked to discriminate two gratings differing slightly in spatial frequency. Our findings indicate that the choice reaction time for spatial frequency discrimination is an inverted U-shaped function of $\log$ spatial frequency. Maximal response latencies occurred near $4 \mathrm{c} / \mathrm{deg}$, where contrast sensitivity is greatest. We assume that this relationship implies that the greater the number of mechanisms involved in the perceptual task, the longer is the computational time required to perform the task successfully. This interpretation suggests that spatial frequency discrimination is, at least in part, a serial process.

\section{METHOD}

Sinewave gratings were produced on a highresolution cathode ray tube (Joyce Electronics, Cambridge, U.K.) under the control of a microprocessor. The mean luminance of the display was $100 \mathrm{~cd} / \mathrm{m}^{2}$. The display subtended $11 \times 15 \mathrm{deg}$ at a viewing distance of $114 \mathrm{~cm}$. A semi-circular, back-illuminated plexiglass screen provided a surround that was approximately matched in luminance and color temperature. The computer controlled the contrast and spatial frequency of the gratings. The grating contrast was turned on and off as a step function of time and the stimulus duration was held constant throughout the experiments at $100 \mathrm{msec}$. A counter-timer integrated circuit was started at the onset of the stimulus, which was used to record the time passing between stimulus onset and the observer's response. The counting circuit was set to have $5 \mathrm{msec}$ resolution.

In the first experiment to be reported, simple reaction time was measured to the onset of a sinewave grating of variable spatial frequency. Six spatial frequencies varying from 0.5 to $16 \mathrm{c} / \mathrm{deg}$ were consecutively presented. An auditory signal cued the subject that the trial was about to begin. After a random delay varying from 0.5 to $1.5 \mathrm{sec}$ the grating was presented and the subject responded by pressing a button. To control for the subject "jumping the gun" or making lapses, the computer was programmed to reject trials that yielded reaction times below $80 \mathrm{msec}$ or above $500 \mathrm{msec}$. Usually no more than 2 trials had to be rejected in a given run. A total of 40 trials for each spatial frequency constituted a run and the average of these recordings was taken as an estimate of reaction time. The data shown in the figures are the average of two or more such runs.

Choice reaction time was measured in a similar way, except now a reference grating of a specified spatial frequency was presented followed by a test grating, the spatial frequency of which was randomly chosen by the computer to be lower or higher than that of the reference grating. The spatial frequency difference between the two gratings was either $0.125,0.25$ or 0.5 octave (corresponding to 9.19 and $41 \%$ ). The absolute contrast and the spatial phase of both reference and test gratings were varied from trial-to trial by a small (maximum $\pm 20 \%$ of base value) random amount to eliminate fixed cues other than the difference in spatial frequency. A random delay, varying from 0.5 to $1.5 \mathrm{sec}$, was now introduced in between the offset of the reference grating and the onset of the test gratings. The observers were asked to press one of two buttons depending on whether the test frequency was higher or lower than the reference spatial frequency. Trials where the subjects made an incorrect judgement or where the reaction time was below $80 \mathrm{mscc}$ or beyond $800 \mathrm{msec}$ were eliminated from the analysis. As before, a total of 40 trials constituted a run and the mean reaction time was calculated from these trials. The average standard error of the mean reaction times was $7.5 \mathrm{msec}$ for simple reaction times and $16-23 \mathrm{msec}$ for choice reaction times varying slightly over subjects and conditions. For choice reaction time measurements, each spatial frequency was measured separately and the order in which the spatial frequencies were tested was randomized. The observers were the authors $M W G$ and $B B$. Both had normal or corrected-to-normal acuity, To eliminate practice effects, training sessions, the results of which were not included in the analysis, were conducted until the reaction time values reached a steady level.

\section{RESULTS}

Simple reaction time as a function of spatial frequency

The results of the first experiment are shown in Fig. 1. Here simple reaction time is plotted as 

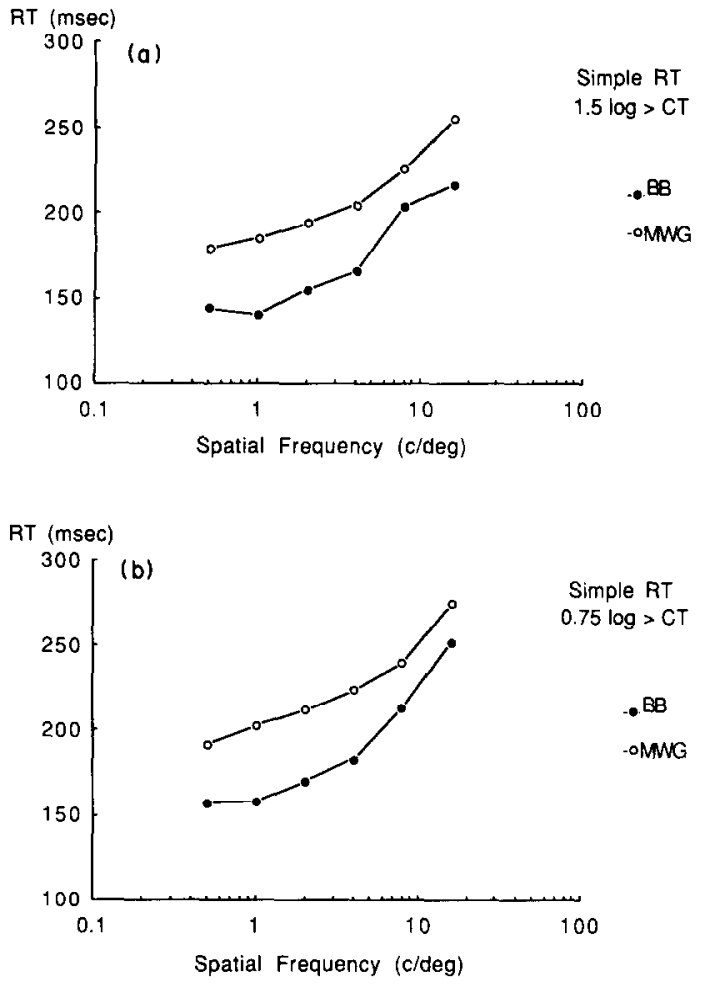

Fig. 1. Simple reaction time (in msec) is plotted as a function of the spatial frequency of sinusoidal gratings. The time passing between the onset of the grating and the subject's response (button press) was recorded by the computer with $5 \mathrm{msec}$ resolution. Symbols show average reaction time. The average standard error over spatial frequencies was $7.2 \mathrm{msec}$ for MWG and $7.8 \mathrm{msec}$ for BB. Results for observer MWG are shown by open symbols, those for BB by solid symbols. Figure la presents the results when grating contrast was $1.5 \log$ units above the detection threshold at the spatial frequency tested and Fig. $1 \mathrm{~b}$ presents similar results for the condition where grating contrast was $0.75 \log$ unit above threshold.

a function of the spatial frequency on linear-log axes. Results for observer MWG are depicted by open symbols, those for observer BB by solid symbols. Figure la shows the results when the grating contrast was $1.5 \mathrm{log}$ units above the respective detection threshold at each spatial frequency and Fig. 1b shows the results when the test grating had a contrast $0.75 \log$ units above threshold. Contrast thresholds were determined in separate experiments using a twointerval forced-choice technique. Detection thresholds did not systematically vary between subjects. We, therefore, took the mean of the threshold values and added the corresponding amount of suprathreshold contrast for the reaction time tasks. The contrast levels used in the experiments were $0.12,0.095,0.063,0.095,0.189$ and 0.4 for spatial frequencies $0.5,1,2,4,8$, and
$16 \mathrm{c} / \mathrm{deg}$, respectively, for the condition in which contrast was $1.5 \log$ units above detection threshold. For the condition using a contrast level $0.75 \mathrm{log}$ unit above threshold, the gratings had a contrast of $0.021,0.017,0.011,0.017$, 0.034 and 0.124 for the same spatial frequencies, respectively.

Averaged over all spatial frequencies, the decrease in grating contrast from 1.5 to $0.75 \mathrm{log}$ units above threshold caused a $14.4 \mathrm{msec}$ increase in reaction time for observer $B B$ and a $17 \mathrm{msec}$ increase for MWG. The original findings of Breitmeyer (1975) are by and large replicated here. For the condition where contrast was $0.75 \mathrm{log}$ unit above threshold, reaction time increases from $160(190)$ at $0.5 \mathrm{c} / \mathrm{deg}$ to 250 (275) $\mathrm{msec}$ at $16 \mathrm{c} / \mathrm{deg}$ for observer BB (values for MWG in brackets). For the condition where contrast was $1.5 \mathrm{log}$ units above detection threshold reaction times increased from 145 (180) to 220 (255) msec for each observer, respectively. Note the large individual differences in reaction time: above $35 \mathrm{msec}$ averaged over spatial frequencies and conditions.

\section{Choice reaction time as a function of spatial} frequency

In this set of experiments we examined the dependency of choice reaction time as a function of the spatial frequency of the reference grating. Figure 2 plots the choice reaction time as a function of reference frequency (on linearlog axes) for the condition where the spatial frequency difference was 0.5 octave. Figure $2 a$ shows the results when the grating contrast was $1.5 \mathrm{log}$ units above threshold and Fig. 2b presents the results when contrast was $0.75 \log$ unit above threshold. The choice reaction time first increases and then decreases with increasing spatial frequency. Maximum reaction times occur at $4 \mathrm{c} / \mathrm{deg}$ for both subjects when contrast was $1.5 \mathrm{log}$ units above threshold and at $2 \mathrm{c} / \mathrm{deg}$ where grating contrast was $0.75 \log$ unit above threshold.

Figure 3 shows similar results, but now the spatial frequency difference between the reference and test gratings was reduced to 0.25 octave. Figure $3 \mathrm{a}$ shows the results for the condition in which grating contrast was adjusted to be $1.5 \log$ units above detection threshold and Fig. $3 b$ presents the results when the gratings were $0.75 \mathrm{log}$ units above threshold. In this condition, choice reaction time peaks between 2 and $4 \mathrm{c} / \mathrm{deg}$ for both subjects tested. Figure 4 presents the results when the spatial 

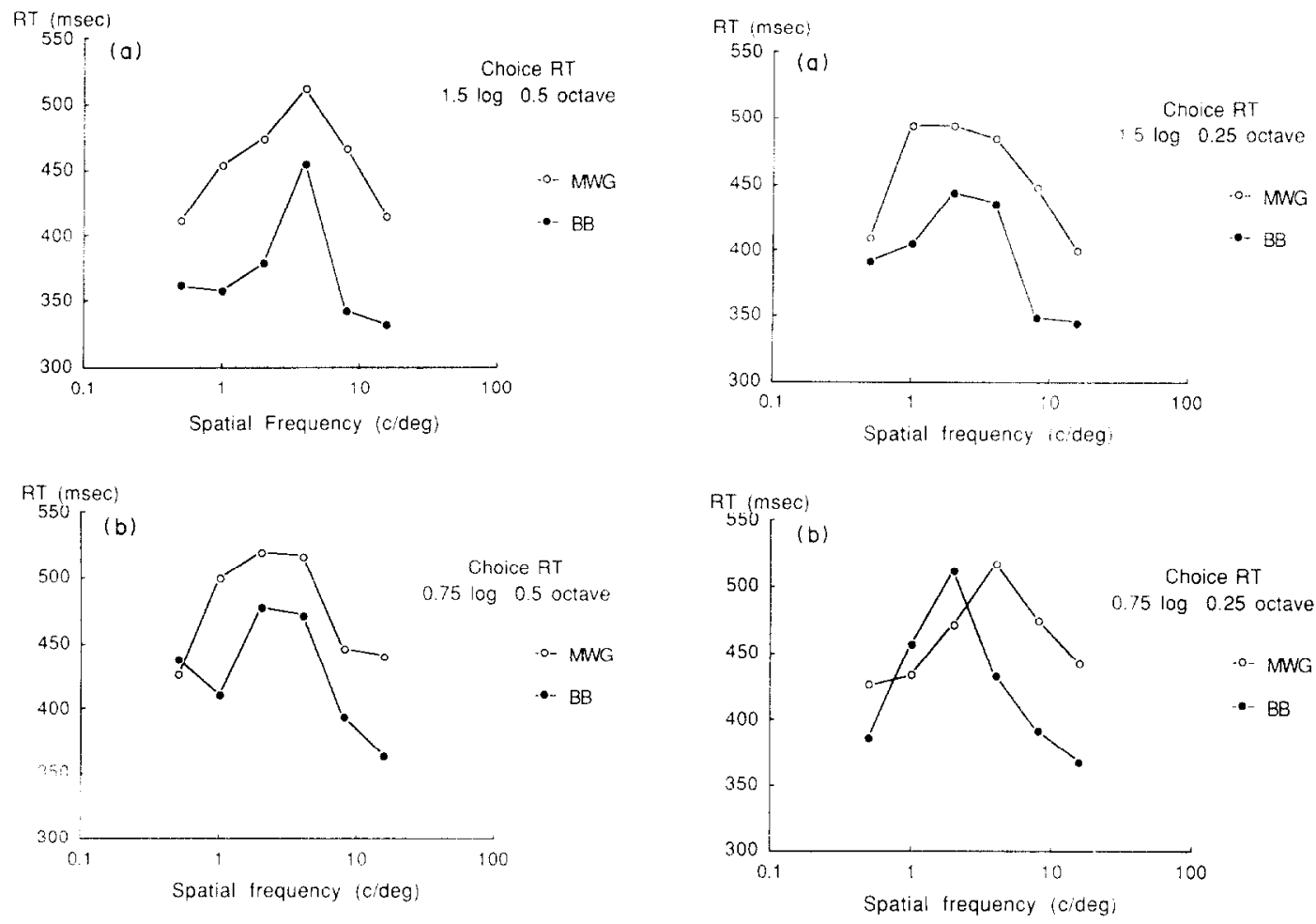

Fig. 2. (hoice reaction time (in msec) for the discrimination of two sinewave gratings differing by 0.5 octave as a function of the spatial frequency of the reference grating. Results for MWG shown by open symbols, those for BB by solid symbols. The average standard error over spatial frequencies was $19.9 \mathrm{sec}$ for MWG and $15.9 \mathrm{msec}$ for RB Figure 2a presents the results when the grating contrast was $1.5 \log$ units above detection threshold, and Fig. 2b shows the results when contrast was $0.75 \mathrm{log}$ unit above threshold.

frequency difference was reduced to 0.125 octave. In this condition, grating contrast was held at $1.5 \mathrm{log}$ units above detection threshold. The lower contrast level proved to be too difficult to get reliable results. As in the results shown in Figs 2 and 3, the choice reaction time first increases and then decreases with spatial frequency. The maximum response latencies occurred in the medium spatial frequency range.

\section{Decision time as a function of spatial frequency}

A simple reaction to grating onset involves, at least, two processes: the sensory and motor process. The sensory process includes the transduction occurring at the retina, the transmission of the action potential along the optic tract, as well as synaptic connections in the lateral geniculate nucleus and in the visual cortex. Differences in response latencies in transient and sustained retinal ganglion cells in the cat have been demonstrated by Bolz, Rosner and Wässle (1982). Evidence for spatial-frequency

Fig. 3. Choice reaction time as a function of spatial frequency for the condition in which the spatial frequency difference was reduced to 0.25 octave. Figure 3 a presents the results when contrast was $1.5 \log$ units above threshold and Fig. $3 b$ shows the results when contrast was $0.75 \log$ unit above threshold. The average standard error over all spatial frequencies was $20.3 \mathrm{msec}$ for MWG and $19.6 \mathrm{msec}$ for BB

dependent delays in the positive component of the human pattern electroretinogram for luminance-contrast, but not color-contrast gratings has been reported by Korth and Rix (1988). Spatial-frequency dependent delays in the visual evoked potential to the onset/offset of

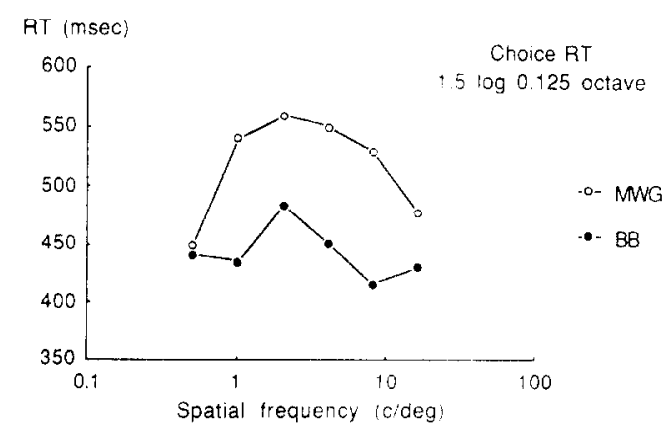

Fig. 4. Choice reaction time as a function of spatial frequency for the condition in which the spatial frequency difference is reduced to 0.125 octave. Only data for the condition where contrast was $1.5 \mathrm{log}$ units above threshold are shown. The average standard error over all spatial frequencies was $22.2 \mathrm{msec}$ for $\mathrm{MWG}$ and $22.8 \mathrm{msec}$ for $\mathrm{BB}$. 
sinewave gratings or to their phase-reversal has been reported by Parker and Salzen (1980), although these latency differences are much smaller than the manual reaction delays (Musselwhite \& Jeffreys, 1985). Together this evidence suggests the existence of difference in the transmission characteristics of neurones in the visual pathway, which could lead to the spatial-frequency dependency in simple reaction time as shown here and in earlier studies. The motor process is thought to be invariant with spatial frequency, although there does seem to be an inhibitory effect of saccadic eye movements occurring directly prior to the manual reaction (Baedcker \& Wolf, 1987).

For a choice reaction, the time required to discriminate the spatial frequency of the two gratings and then to correctly respond involves, at least, one further process. This process compares the most recent sensory input (elicited by the second grating) to that which occurred some $500-1500$ msec earlier (elicited by the first grating). This process is more than just sensory in nature since the most recent visual input has to be compared to the memory trace of the earlier input. It could best be thought of as a cognitive event, since a decision has to be made as to which button is to be pressed based on the sign of the perceived spatial frequency difference. We can derive the decision time in a given condition by subtracting the simple reaction time from the choice reaction time at the reference spatial frequency in question. We do not intend to imply here that performance of the simple reaction time task does not involve cognitive processes. Of course, the observer must decide when the grating is presented in a simple reaction time task, but no decision is required concerning the relative spatial frequency of the grating.

The results of this analysis are shown for two conditions in Fig. 5. Figure 5a plots the reaction time difference, which we will refer to as the decision time, as a function of spatial frequency when the grating contrast was $1.5 \log$ units above threshold and the spatial frequency difference was 0.5 octave. Figure $5 \mathrm{~b}$ shows similar results, when the spatial frequency difference between the first and second grating was reduced to 0.25 octave. Average decision times first increase with spatial frequency up to the medium frequency range (between 1 and $4 \mathrm{c} / \mathrm{deg}$ ) and then decrease again at high spatial frequencies. In Fig. 5a, for subject BB a $0.5 \mathrm{c} / \mathrm{deg}$ grating could be discriminated from a 0.35 or a $0.7 \mathrm{c} / \mathrm{deg}$ grating in an average of
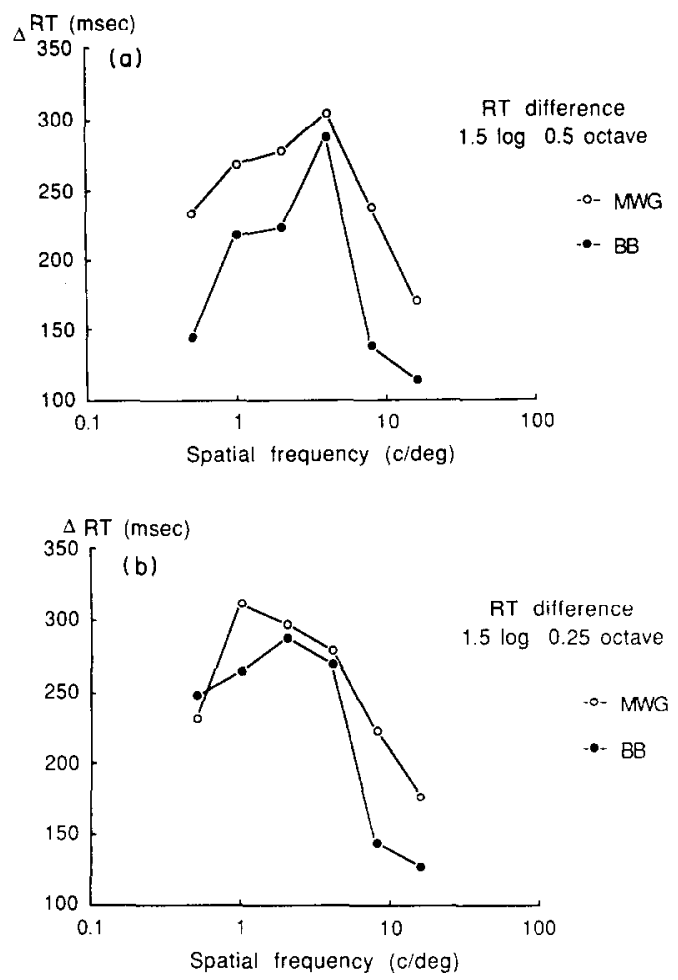

Fig. 5. The decision time required by the subjects to perform the spatial frequency discrimination task (RT choice - RT simple) is shown as a function of spatial frequency. Grating contrast was $1.5 \mathrm{log}$ units above detection threshold. Figure $5 a$ presents the results when the spatial frequency of the gratings differed by 0.5 octaves and Fig. 5 b shows the results when the spatial frequency of the gratings differed by 0.25 octaves.

$140 \mathrm{msec}$, whereas the 0.5 octave discrimination around a reference frequency of $4 \mathrm{c} / \mathrm{deg}$ grating required twice as long $(280 \mathrm{msec})$. A $16 \mathrm{c} / \mathrm{deg}$ grating, on the other hand could be discriminated from a test grating differing by 0.5 octave in as little as $110 \mathrm{msec}$. Similar trends were found when the gratings differed by 0.25 octave. Although requiring slightly more decision time, observer MWG produced reaction times showing the same dependency on spatial frequency.

\section{Effect of spatial-frequency difference on choice reaction time}

To analyze how the spatial frequency difference affects the decision time, we plotted the decision time for each subject averaged over all spatial frequencies tested as a function of the spatial frequency difference. The results of this analysis are shown in Fig. 6. The grating contrast used for this comparison was $1.5 \log$ units above detection threshold. The decision time decreases with increasing spatial frequency difference. When the spatial frequency of the gratings differs by only 0.125 octave, the time 


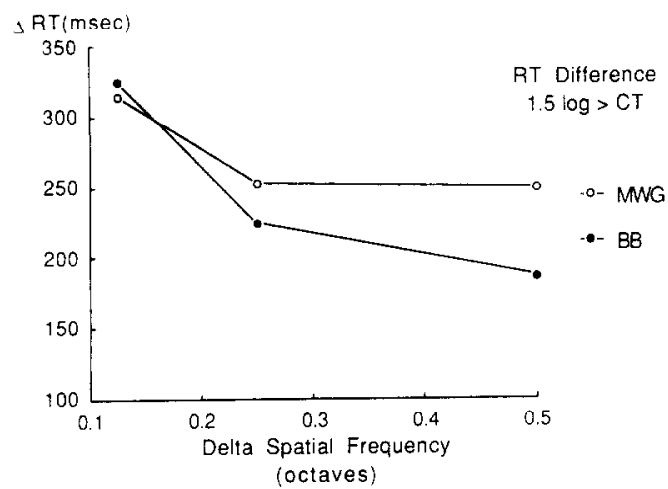

Fig. 6. The decision time ( $\mathrm{RT}$ choice - RT simple), averaged over all spatial frequencies tested, is shown as a function of the spatial difference (in octaves)

required for the subject to make a decision is, on average, $320 \mathrm{msec}$. Increasing the spatial frequency difference to 0.5 octave decreases the decision time to $218 \mathrm{msec}$ averaged over subjects. Thus about a $100 \mathrm{msec}$ reduction in average decision time occurs when increasing the spatial frequency difference from 0.125 to 0.5 octaves.

Effect of grating contrast on simple and choice reaction time

The contrast of the reference and test gratings used in the reaction time experiments reported above was adjusted for each spatial frequency to remain a constant amount (in logarithmic units) above detection threshold. It is well known, however, that the apparent contrast of gratings varying in spatial frequency but having the same high physical contrast level appears constant, a phenomenon referred to as contrast constancy (Georgeson \& Sullivan, 1975). It could thus be argued that the inverted $\mathrm{u}$-shaped functions of choice reaction time vs spatial frequency (Figs 2-4) could be related to the different contrast levels of the gratings. For medium spatial frequencies between $2-4 \mathrm{c} / \mathrm{deg}$, sensitivity is greatest and thus the gratings used in the reaction time tasks had the lowest contrast at those frequencies. Choice reaction to gratings having a constant suprathreshold contrast level would, according to this line of reasoning, covary with the contrast sensitivity function. The results could thus be related to an effect of the physical contrast of the gratings and not to their spatial frequency per se.

To control for this possibility, we conducted a further experiment in which we explored the effect of grating contrast on simple and choice reaction time. Grating contrast was varied from
0.25 to $2.0 \mathrm{log}$ units above threshold. Two base spatial frequencies were used: 0.5 and $4.0 \mathrm{c} / \mathrm{deg}$. All other conditions were the same as those described in the Method section. Figure 7 presents the results of this experiment for observer MWG. Simple reaction time is plotted as a function of the suprathreshold contrast level (Fig. 7a) or the physical contrast (Fig. 7b) of the test gratings having a low (solid symbols) or intermediate (open symbols) spatial frequency. In agreement with the earlier findings of Harwerth and Levi (1978), simple reaction time decreases with increasing suprathreshold contrast. Reaction times level off between 1 and $1.5 \log$ units above threshold. In terms of physical grating contrast, reaction times increase rapidly when grating contrast is reduced below $5 \%$ (Fig. $7 b$ ).

Choice reaction time is shown in Fig. 8, again either as a function of log suprathreshold contrast (a) or the physical contrast of the gratings
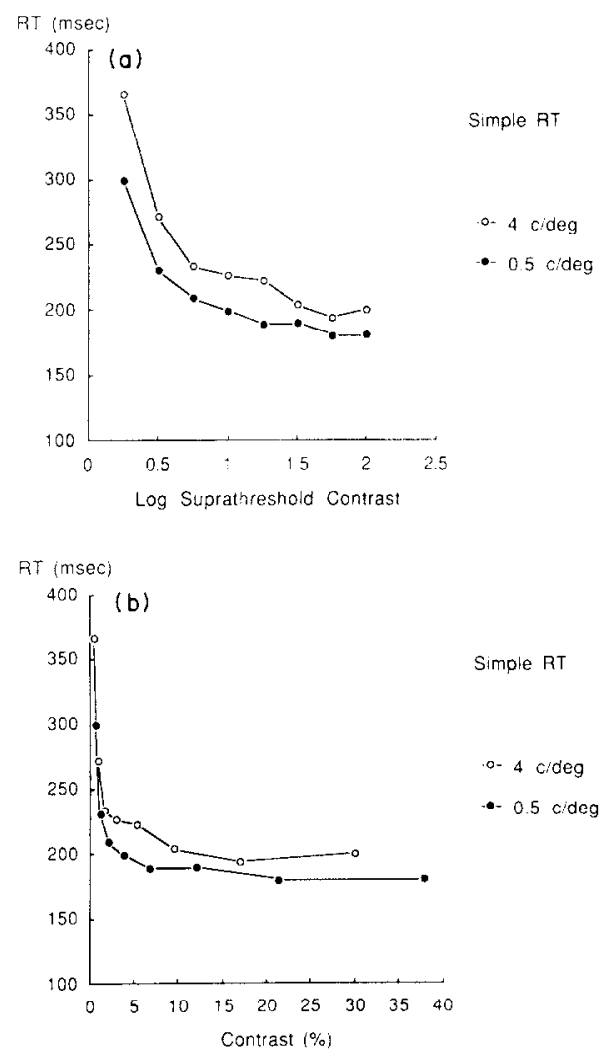

Fig. 7. Simple reaction time as a function of grating contrast. In (a) simple reaction times are plotted as a function of log suprathreshold contrast, whereas in (b) reaction time is shown as a function of grating contrast on a linear scale. Open circles present the results for the $4 \mathrm{c} / \mathrm{deg}$ grating and solid circles show the findings of the $0.5 \mathrm{c} / \mathrm{deg}$ grating. The symbols represent the mean of 3 runs. Results shown for observer MWG. 

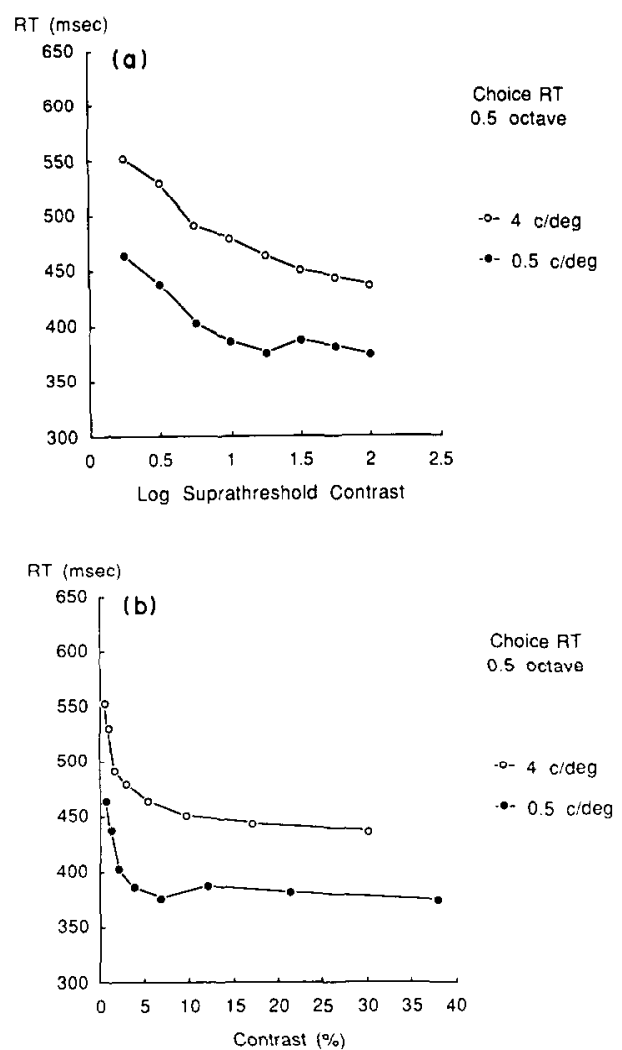

Fig. 8. Choice reaction time as a function of grating contrast. In (a) choice reaction times are plotted as a function of $\log$ suprathreshold contrast, whereas in (b) reaction time is shown as a function of grating contrast on a linear scale. Open circles present the results for the $4 \mathrm{c} / \mathrm{deg}$ grating and solid circles show the findings of the $0.5 \mathrm{c} / \mathrm{deg}$ grating. The symbols represent the mean of 3 runs. Results shown for observer MWG.

(b). The spatial frequency difference in the choice reaction task was 0.5 octave. For both spatial frequencies tested, choice reaction time decreases with increasing suprathreshold contrast. For example, in the $0.5 \mathrm{c} / \mathrm{deg}$ condition, reaction time decreases from 464 to $374 \mathrm{msec}$ when suprathreshold contrast is increased from 0.25 to $2.0 \mathrm{log}$ units. This is a change of $90 \mathrm{msec}$. The difference in reactions times for the low and medium spatial frequency conditions remains, however, fairly constant at $80 \mathrm{msec} \pm 14 \mathrm{msec}$ for the contrast range tested. The two curves remain clearly separated when choice reaction time is plotted as a function of physical contrast (Fig. 8b). It follows that, although there is a clear dependency between choice reaction time and contrast (up to $10 \%$ contrast), the nonmonotonic nature of the choice reaction time vs spatial frequency function cannot be explained as an artifact of the differences in the apparent or real contrast levels of the gratings.
More important for our discussion here is the effect of contrast on the time required by the observer to make a correct decision with regard to the relative spatial frequency of the two gratings. The results of this analysis are shown in Fig. 9, in which the difference between the choice and simple reaction times are plotted either as a function of the log suprathreshold contrast (Fig. 9a) or as a function of (linear) grating contrast (Fig. 9b). The results indicate that the decision time remains fairly constant for a large range of grating contrast values. However, when grating contrast approaches the contrast threshold for the respective spatial frequency, the decision time suddenly decreases and the difference beween the low and intermediate spatial frequency also decreases.

\section{DISCUSSION}

\section{Simple reaction time and spatial frequency}

The results of our first experiment confirm the earlier findings of Breitmeyer (1975) that perceptual latency increases monotonically with spatial frequency. There are, however, some

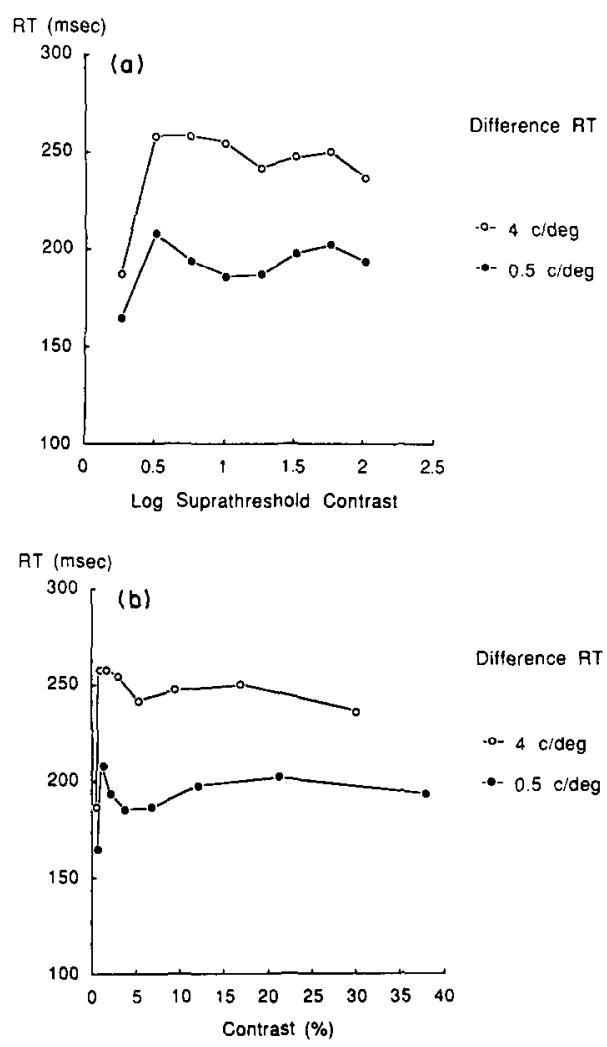

Fig. 9. The decision time required for the observer to make a correct spatial-frequency discrimination ( $R T$ choice $-R T$ simple, values from Figs 7 and 8 ) is shown as a function of $\log$ suprathreshold contrast (a) or as a function of physical grating contrast on a linear scale (b). 
differences between our experimental conditions and those of the earlier studies. Breitmeyer (1975, experiment 2) had his subjects match the contrast of the test grating at different spatial frequencies to that of a $11 \mathrm{c} / \mathrm{deg}$ grating of 0.66 contrast. The grating exposure time in his experiment was $50 \mathrm{msec}$. Observer BB's contrast threshold (measured in separate experiments) for this exposure duration and spatial frequency is approx. 0.025 contrast. With a grating contrast of 0.66 , this would be equivalent to a $1.42 \log$ unit increase above his contrast threshold, and is thus close to our condition of $1.5 \mathrm{log}$ units above threshold. Unfortunately Breitmeyer was not a subject in his own original experiment, since we could have had the rare opportunity of comparing his reaction time after more than 13 years of life. However, comparison of subject BB's results (Fig. 1a) and those of Breitmeyer's subject KA in his Fig. 2 indicates a remarkable similarity. Observer KA showed a reaction time of $175 \mathrm{msec}$ for a $0.5 \mathrm{c} / \mathrm{deg}$ grating and a $205 \mathrm{msec}$ reaction time for a $11 \mathrm{c} / \mathrm{deg}$ grating. In our experiment, BB responded, on average, $144 \mathrm{msec}$ after the onset of a $0.5 \mathrm{c} / \mathrm{deg}$ grating and $217 \mathrm{msec}$ after the onset of a $16 \mathrm{c} / \mathrm{deg}$ grating $(11 \mathrm{c} / \mathrm{deg}$ was not measured in the present study). Considering the differences in laboratories, subjects, and time this similarity is truly remarkable. Thus the dependence of simple reaction on spatial frequency can be thought of as a replicable finding (cf. Lupp et al., 1976; Vassilev \& Mitov, 1976; Harwerth \& Levi, 1978). The correlation between this perceptual latency and possible neurophysiological differences in transmission delays between transient and sustained pathways in the primate visual system, however, remains to be clarified (Musselwhite \& Jefferies, 1985; Baedeker \& Wolf, 1987).

\section{Choice reaction time and spatial frequency}

Contrary to simple reaction time, the choice reaction time of spatial frequency discrimination is not a monotonic function of spatial frequency. As evident in Figs 2-5, the time required by the subjects to correctly discriminate the spatial frequency of the two gratings first increases with spatial frequency and then decreases with a further increase in spatial frequency. The maximum choice reaction time usually occurred between 2 and $4 \mathrm{c} / \mathrm{deg}$ varying between subjects and conditions. Since the choice reaction time reflects the same processes involved in simple reaction time plus the deci- sion process of the spatial frequency discrimination task, we could derive the mean decision time required by the subjects to successfully perform the discrimination task and plot this value as a function of spatial frequency. The results of this analysis also revealed that decision time first increases and then decreases with spatial frequency (Fig. 5). In our experimental design, precautions were taken so that the subjects could not use other cues besides the spatial frequency difference, which might have otherwise eased the task at low and high spatial frequencies, and could thus have been responsible for the shape of these functions. For example, we varied the contrast of each grating from presentation-to-presentation by a random constant so that relative differences in the perceived contrast of the reference and test gratings became an unreliable cue. The spatial phase of the grating was also varied for each grating before onset, so that the position of the gratings period with respect to the fixation point could not be used as a cue. Such a cue could have proved beneficial especially for low spatial frequencies. We conclude that our results cannot be accounted for by some sort of additional information that occurs when varying the spatial frequency of a grating.

\section{Effect of grating contrast on reaction time}

When we explored the effect of grating contrast on the simple and choice reaction time we found that reaction time decreased with increasing suprathreshold contrast (Figs 7 and 8). Differences in reaction time between the low and medium spatial frequency conditions remained, however, fairly invariant of contrast, with an average latency difference of approx. $80 \mathrm{msec}$. The spatial-frequency dependent reaction time differences cannot, therefore, be accounted for by differences in the physical contrast of the test gratings used in the experiments.

Interestingly, it has earlier been shown (Hirsch \& Hylton, 1982) that the Weber fraction for spatial frequency discrimination of gratings with spatial frequencies near $4 \mathrm{c} / \mathrm{deg}$ is slightly larger than for higher or lower spatial frequencies. This contradicts first expectation, since the contrast sensitivity function peaks in this spatial frequency range and it is often assumed that other aspects of vision might also be best for these spatial frequencies. For a contrast detection task, it may be assumed that threshold is in some way inversely related to the density of receptive fields that have a preferred spatial 
frequency at or near the spatial frequency of the grating tested. If this assumption is true, then the question arises why the spatial frequency discrimination threshold should be slightly worse at these frequencies, and, as the present findings show, choice reaction times are more prolonged. Mayer and Kim (1986) however, were unable to replicate the irregularities in the spatial-frequency discrimination function reported by Hirsch and Hylton (1982). To understand these apparently conflicting observations a model for spatial frequency discrimination needs to be proposed. We would like next to give a qualitative description of what such a model may look like.

\section{Spatial frequency discrimination $-a$ serial process?}

The spatial frequency discrimination performed by the human visual system has been conceptualized by, at least, two different approaches. The first approach is based on a multi-channel analysis of the spatial frequency content of the two gratings (e.g. Campbell et al., 1970), where the performance of the visual system can be thought of in terms of a sizeopponent (Regan \& Beverley, 1983) or a modified line-element model (Wilson \& Gelb, 1984; Wilson \& Regan, 1984). In the Wilson and Gelb (1984) model, the pooled responses of six channells responding to the first pattern would be compared to the pooled response distribution evoked in the same channels responding to the second pattern. The spatial discrimination threshold would thus depend on the bandwidth and spatial-frequency separation of the underlying neural channels, the gain of each channel, and on the suprathreshold contrast level of the grating. With two free parameters, one determining the summation rule among channels and another depicting the distance over which spatially adjacent neighbouring channels are pooled, Wilson and Gelb (1984) could successfully predict the positions of the peaks and troughs in the spatial-frequency discrimination function described by Hirsch and Hylton (1982).

The second approach is based on the local feature analysis of the distance between two adjacent peaks or troughs in the grating (Hirsch \& Hylton, 1982). In this model the resolution of the neural lattice is the limiting factor and the discrimination threshold vs spatial frequency function can be described by a segmentation rule, where a constant separation accuracy is maintained for a given level of resolution. Both of these approaches share a common parsimonious rule of using as few channels (or scales) as possible to model human visual performance.

Our present findings indicate that the decision time required to perform the discrimination task is a nonmonotonic function of spatial frequency, choice reaction time at intermediate spatial frequencies being a factor of 2 or greater than at low or high spatial frequencies. This finding does not easily fit into the Hirsch and Hylton (1982) scaled-lattice model. If spatial frequency discrimination were based on a localized feature analysis that measures the peak-topeak separation of the grating's period, then the lower the spatial frequency the more distant would become the peaks and the greater would be the number of mechanisms in space that would be required for the comparison. Such a process should yield a monotonically decreasing function of decision time versus spatial frequency if these mechanisms were compared serially or it would yield a flat function if the mechanisms were processed in parallel. Interestingly, Hirsch and Hylton (1982) specifically excluded spatial frequencies of $2 \mathrm{c} / \mathrm{deg}$ and below from their original model, since their model predicted lower thresholds than those actually observed. In a later study, Hirsch and Hylton (1985) applied a segmentation rule based on the size of retinal receptive fields and not the spacing of the foveal cones to account for discrimination threshold between 0.3 and $2 \mathrm{c} / \mathrm{deg}$.

The present findings may be more compatible with a multi-channel model based on a discrete number of spatial-frequency channels (Wilson \& Gelb, 1984). There is evidence from masking experiments (Wilson, McFarlane \& Phillips, 1983) that the "peak" spatial frequencies of the channels may not be evenly spaced in the frequency domain. If channels responding best to medium spatial frequencies are more dense in the frequency domain and thus their response profiles would have greater overlap than those for low or high spatial frequencies, the number of pairwise comparisons required among the active channels would be grcatest in the medium spatial frequency range. Such an array of channels and their overlap in the spatial frequency domain are depicted schematically in Fig. 10. The circles represent the spatial-frequency passband of channels centered at specific spatial frequencies have full bandwidths of about 1 octave (corresponding to the diameter of the circles). The peak spatial frequencies of the 


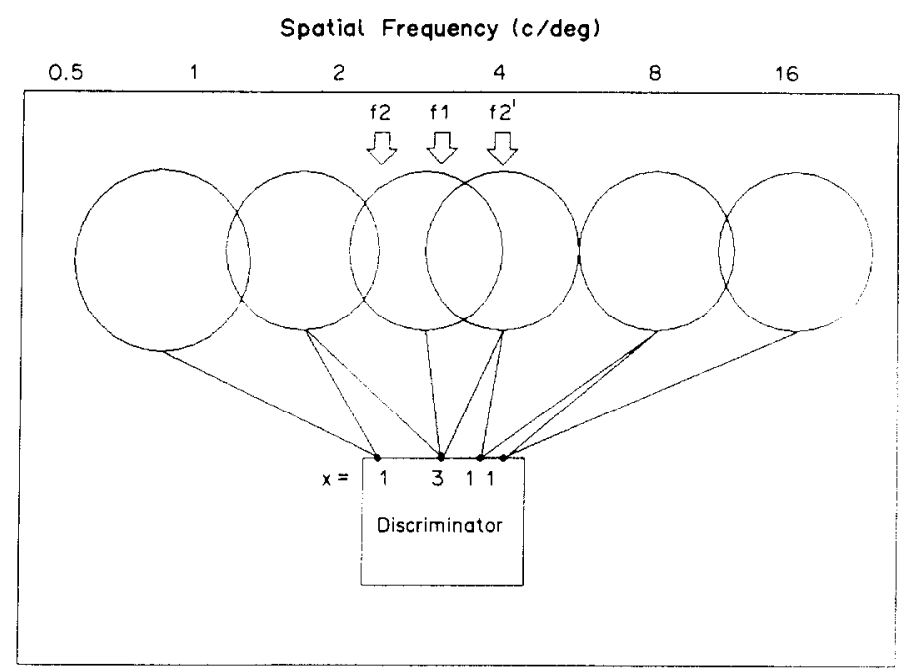

Fig. 10. A schematic representation of a spatial frequency discriminator. The circles represent the spatial-frequency selectivity (passband) of the receptive fields of neurons in the visual cortex. The number and peak frequencies of the channels depicted have been taken from Wilson and Gelb (1984, their Table 1). The discriminator is some higher-order neural process which receives input from all receptive fields at a given retinal locus. The decision nodes (represented by the common points on the discriminator box) indicate the number of mechanisms that are compared for a given spatial-frequency range. The numbers inside the discriminator indicate the number of pairwise comparisons that would have to be made with the number of inputs shown at each node.

6-channel model presented by Wilson and Gelb (1984) have been chosen as an example. We do not want to imply that our data support or reject the Wilson and Gelb model. We rather use it to illustrate how the information from different spatial-frequency channels could be compared.

In this scheme, spatial frequency discrimination is based on the pairwise comparison of channel responses at a given node at the hypothetical discriminator. Such a discriminator could be any higher-order process that serially compares the outputs of each of the connected channels. The greater the overlap (i.e. density in the spatial frequency domain) of these mechanisms, the larger the number of pairwise comparisons that have to be made. This is depicted by the number of channels connected to a given node at the discriminator. Since the number of pairwise comparisons increases with $(x(x-1)) / 2$, a change from $x=2$ to $x=3$ channels leads to an increase of 1 to 3 pairwise comparisons, and would thus, in a serial process, approximately increase the computational time required by a factor of three. For low or high spatial frequencies, the channel overlap is less and thus the number of possible comparisons decreases. This would decrease the response latency, assuming that the frequency discrimination process occurs serially. In the medium spatial frequency range (between 2 and $4 \mathrm{c} / \mathrm{deg}$ ) the number of mechanisms and their overlap in the frequency domain is high. This greater channel density increases the number of comparisons that have be made made and thus increases the response latency. Although the model is purely descriptive and ad hoc, it would explain the increased response latency for spatial-frequency discriminations at intermediate spatial frequencies found here.

\section{Grating contrast and decision uncertainty}

The findings in Figs 7 and 8 indicate that response latency decreases with increasing suprathreshold stimulus contrast. At first glance this finding appears to contradict the "channeldensity" hypothesis, since the lower the grating contrast the lower should be the number of active detectors. Above $10 \%$ contrast, choice reaction times becomes independent of test contrast. Thus for low contrast stimuli (i.e. below $10 \%$ ), an additional factor appears to play a role in determining choice reaction time in the spatial-frequency discrimination task. This factor could be related to stimulus uncertainty (Pelli, 1985; Kramer, Graham \& Yager, 1985). We assume that the lower the stimulus contrast the greater is the uncertainty regarding (1) the 
time of stimulus onset and (2) the spatial frequency of the grating (or, as required in our task, the sign of the spatial-frequency difference). Both of these factors would affect the choice reaction time. It has earlier been shown (Watson \& Robson, 1981; Thomas, 1983), that the spatial-frequency discrimination threshold increases when test contrast approaches detcction threshold. Such a relationship suggests an increase in stimulus uncertainty at low contrast levels. As mentioned above, the choice-reaction time difference between the low and intermediate spatial frequencies remained fairly constant over the contrast range tested. Interestingly, however, when we subtracted the simple from choice reaction times for each contrast level and spatial frequency, we found that decision time remained fairly independent of grating contrast, until contrast was reduced to near threshold values (Fig. 9). At values near threshold (0.25 log unit suprathreshold) reaction times suddenly decreased. For the $4 \mathrm{c} / \mathrm{deg}$ grating this decrease corresponded to $70 \mathrm{msec}$. As our error of measurement was approximately a factor of 4 less than this, this difference can be thought of as reflecting a real change and cannot be accounted for by chance fluctuations. We suggest that the additional response latency at intermediate spatial frequencies is caused by the larger number of channels activated at those frequencies. By decreasing the grating contrast to nearthreshold values we reduce the number of activated channels and, as a consequence, the decision time is reduced. But as we know from earlier studies (e.g. Thomas, 1983), the accuracy of the spatial-frequency judgement is also reduced at near-threshold contrast levels.

In summary, the results of the present experiments indicate that choice reaction time is a nonmonotonic function of spatial frequency. The observers require more time to solve the discrimination task for gratings of $2-4 \mathrm{c} / \mathrm{deg}$ than for gratings of lower or higher spatial frequencies. Such a delay could reflect the density in the spatial frequency domain of sizeselective mechanisms, the greatest density occurring near $4 \mathrm{c} / \mathrm{deg}$ in central vision. The results also suggest that the spatial frequency discrimination of two gratings is, at least in part, a serial process.

Acknowledgements - This research was supported by the Deutsche Forschungsgemeinschaft (SFB 325, B4) and the Alexander von Humboldt Foundation (Bonn). The authors thank L. Spillmann and S. Magnussen for providing helpful comments at an initial stage of this work.

\section{REFERENCES}

Baedeker, C. \& Wolf, W. (1987). Influence of saccades on manual reactions - a reaction time and VEP study. Vision Research, 27, 609-619.

Bolz, J., Rosner, G. \& Wässle, H. (1982). Response latency of brisk-sustained (X) and brisk-transient $(\mathrm{Y})$ cells in the cat retina. Journal of Physiology, London, 328, 171-190.

Breitmeyer, B. G. (1975). Simple reaction time as a measure of the temporal response properties of transient and sustained channels. Vision Research, 15, 1411-1412.

Breitmeyer, B. G. (1984). Visual masking: An integrative approach. London: Oxford University Press.

Campbell, F. W., Nachmias, J. \& Jukes, J. (1970). Spatialfrequency discrimination in human vision. Journal of the Optical Society of America, 60, 555-559.

Georgeson, M. A. \& Sullivan, G. D. (1975). Contrast constancy: Deblurring in human vision by spatial frequency channels. Journal of Physiology, London, 252, $627-656$

Harwerth, R. S. \& Levi, D. M. (1978). Reaction time as a measure of suprathreshold grating detection. Vision Research, 18, 1579-1586.

Hirsch, J. \& Hylton, R. (1982). Limits of spatial frequency difference thresholds as evidence of neural interpolation. Journal of the Optical Society of America, 72, 1367-1374.

Hirsch, J. \& Hylton, R. (1985). Spatial-frequency discrimination at low frequencies: Evidence for position quantization by receptive fields. Journal of the Optical Society of America, A 2, 128-135.

Johnson, H. M. (1923). Reaction time measurements. Psychological Bulletin, 20, 562-589.

Kramer, P., Graham, N. \& Yager, D. (1985). Simultaneous measurement of spatial-frequency summation and uncertainty effects. Journal of the Optical Society of America, A 2, 1533-1542.

Korth, M. \& Rix, R. (1988). Luminance-contrast evoked responses and color-contrast evoked responses in the human electroretinogram. Vision Research, 28, 41-48.

Mayer, M. J. \& Kim, C. B. (1986). Smooth frequency discrimination functions for foveal, high-contrast, mid spatial frequencies. Journal of the Optical Society of America, A 3, 1957-1969.

Lupp, U., Hauske, G. \& Wolf, W. (1976). Perceptual latencies to sinusoidal gratings. Vision Research, 16, 969-972.

Musselwhite, M. J. \& Jeffreys, D. A. (1985). The influence of spatial frequency on the reaction times and evoked potentials recorded to grating pattern stimuli. Vision Research, 25, 1545-1555.

Parker, D. M. \& Salzen, E. A. (1980). Evoked potentials and reaction times to the offset and contrast reversal of sinusoidal gratings. Vision Research, 22, 205-207.

Pelli, D. G. (1985). Uncertainty explains many aspects of visual contrast detection and discrimination. Journal of the Optical Society of America, A 2, 1508-1532.

Regan, D. \& Beverley, K. I. (1983). Spatial frequency discrimination and detection: Comparison of postadaptation thresholds. Journal of the Optical Society of America, $73,1684-1690$

Rudd, M. E. (1988). Quantal fluctuation limitations on reaction time to sinusoidal gratings. Vision Research, 28 , 179-186.

Teichner, W. H. (1953). Recent studies of simple reaction time. Psychological Bulletin, 5I, 128-149. 
Thomas, J. P. (1983). Underlying psychometric function for detecting gratings and identifying spatial frequency. Journal of the Optical Society of America, 73, 751-758.

Vassilev, A. \& Mitov, D. (1976). Perception time and spatial frequency. Vision Research, 16, 89-92.

Watson, A. B. \& Robson, J. G. (1981). Discrimination at threshold: Labelled detectors in human vision. Vision Research, 2l, 1115-1122.

Wilson, H. R. \& Gelb, D. J. (1984). Modified line-element theory of spatial-frequency and width discrimination. Journal of the Optical Society of America A, 1, 124-131.
Wilson, H. R., McFarlane, D. K. \& Phillips, G. C. (1983). Spatial frequency tuning of orientation selective units estimated by oblique masking. Vision Research, 23, 873-882.

Wilson, H. R. \& Regan, D. (1984). Spatial-frequency adaptation and grating discrimination: Predictions of a lineelement model. Journal of the Optical Society of America A 1, 1091-1096.

Yager, D. \& Richter, E. (1982) Spatial frequency difference thresholds are not monotonic with frequency. Investigative Ophthalmology and Visual Science, 22, 251 (Suppl.). 